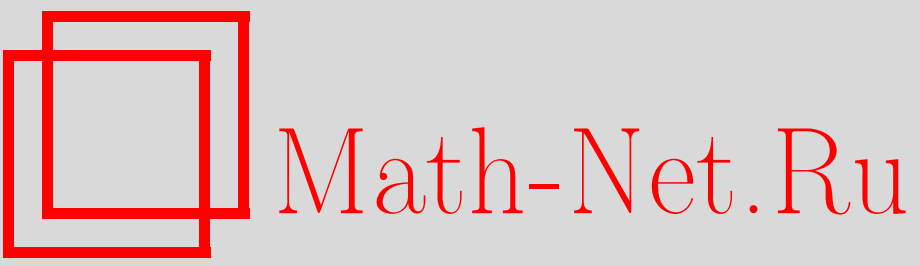

Н. Г. Мазур, Квазиклассическая асимптотика в методе обратной задачи рассеяния для уравнений КдФ и решение модуляционных уравнений Уизема, ТМФ, 1996, том 106, номер 1, 44-61

DOI: https://doi.org/10.4213/tmf1096

Использование Общероссийского математического портала Math-Net.Ru подразумевает, что вы прочитали и согласны с пользовательским соглашением

http: //www . mathnet.ru/rus/agreement

Параметры загрузки:

IP: 54.237 .206 .68

26 апреля 2023 г., 11:17:03 


\section{КВАЗИКЛАССИЧЕСКАЯ АСИМПТОТИКА В МЕТОДЕ ОБРАТНОЙ ЗАДАЧИ РАССЕЯНИЯ ДЛЯ УРАВНЕНИЙ КДФ И РЕШЕНИЕ МОДУЛЯЦИОННЫХ УРАВНЕНИЙ УИЗЕМА}

Исследована задача Коши для уравнения Кортевега-де Фриза в слабодисперсионном пределе, моделирующая образование и эволюцию бездиссипативной ударной волны в плазме. Теория возмущений по малому параметру при дисперсионном члене приводит к уравнению простой волны, а после опрокидывания простой волны - к системе модуляционных уравнений Уизема, описываюшей возникшую бездиссипативную ударную волну. В работе использован альтернативный подход, состоящий в асимптотическом исследовании в пределе слабой дисперсии точного решения, полученного методом обратной задачи рассеяния. При этом используются формулы квазиклассики в прямой задаче рассеяния и точное многосолитонное решение в обратной задаче. В результате перехода к пределу получена система конечных соотношений, связывающих $x, t$ и модуляционные параметры бездиссипативной ударной волны.

\section{1. ВВЕДЕНИЕ}

Задача Коши для уравнения КдФ

$$
\begin{gathered}
u_{t}-6 u u_{x}+\varepsilon^{2} u_{x x x}=0, \\
u(x, 0)=u_{0}(x)
\end{gathered}
$$

в слабодисперсионном пределе $\varepsilon \rightarrow 0$ может изучаться с помошью двух подходов.

Первый подход, основанный на теории возмушений с применением многомасштабных разложений, развит в работах [1-10]. Он приводит для описания начального этапа эволюции к уравнению Хопфа

$$
u_{t}-6 u u_{x}=0,
$$

решение которого годится только до момента опрокидывания $t_{*}$. При $t>t_{*}$ появляется расширяюшаяся со временем область, заполненная модулированным пакетом нелинейных (кноидальных) волн длины $\sim \varepsilon$. В этой области ситуация описывается модуляшионной теорией Уизема [1]. В простейшем и наиболее типичном случае однофазного волнового пакета система уравнений Уизема состоит из трех квазилинейных уравнений первого порядка; она имеет диагональную риманову форму. Впервые задача о 
сшивке решения уравнений Уизема с "внешним" решением уравнения Хопфа поставлена в [2] (задача Гуревича-Питаевского); там же получены важные автомодельные решения, описываюшие распад начального разрыва и начальную стадию после момента опрокидьвания. Более обшие решения этой задачи были найдены в работах $[3,4]$, где с применением метода годографа исследован случай, когда из трех римановых переменных, подчиняюшихся уравнениям Уизема, одна является постоянной, а остальные две искомыми величинами. Наконец, на основе обобщенного метода годографа $[5,6]$ было построено общее аналитическое решение задачи Гуревича-Питаевского [7-11].

Второй подход состоит в изучении асимптотики при $\varepsilon \rightarrow 0$ точного решения задачи (1), (2) методом обратной задачи рассеяния (МОЗР). Сушественный прогресс в этом направлении достигнут в серии работ Лакса и Левермора [12], где путем прямого асимптотического исследования при $\varepsilon \rightarrow 0$ формул ОЗР получено уравнение (3) при $t \leq t_{*}$, а при $t>t_{*}-$ уравнения Уизема (и их многофазное обобщение). Метод Лакса-Левермора был расширен Венакидесом $[13,14]$ на случай непрерывного спектра (ненулевого коэффициента отражения).Бикбаев $[15,16]$ использовал традиционный подход МОЗР с нетривиальными граничными условиями на бесконечности. В работе Геогджаева [17] изучен квазиклассический предельный вариант МОЗР для уравнения КдФ и нелинейного уравнения Шредингера.

Суть второго подхода заключается в применении формул квазиклассического приближения (ВКБ) для решения прямой задачи рассеяния при $t=0$, т.е. на потенциале $u_{0}(x)$. Далее, обратная задача рассеяния при $t>0$ может быть решена путем обрашения этих формул, но только для $t \leq t_{*}$. Для бо́льших времен приходится решать обратную задачу точно, т.е. с помощью уравнения Гельфанда-Левитана. Однако сильное упрошение происходит при $\varepsilon \rightarrow 0$ и в случае $t>t_{*}$ благодаря тому, что коэффициент отражения $r(k ; \varepsilon) \rightarrow 0$, так что в ядре уравнения Гельфанда-Левитана можно пренебречь частью, отвечаюшей непрерывному спектру. Это позволяет заменить точное решение задачи (1), (2) безотражательным потенциалом, эволюционируюшим согласно уравнению (1), т.е. $N$-солитонным решением $\left(N \sim \varepsilon^{-1} \rightarrow \infty\right)$, выражающимся известной явной элементарной формулой. Непосредственное исследование этого выражения при $N \rightarrow \infty$ и решает поставленную задачу, приводя к результатам, совпадающим с полученными методом теории возмущений.

Заметим, что хотя при $t \leq t_{*}$ использование многосолитонной формулы для решения обратной задачи рассеяния не является необходимым, тем не менее она, разумеется, работает и в этом случае и дает тот же ответ (при $N \rightarrow \infty$ ), что и обрашение ВКБ-формул прямой задачи.

Наиболее интересен в связи с задачей (1), (2) вопрос о качественном характере механизма возникновения осцилляционной зоны после момента опрокидывания $t_{*}$. Это явление можно рассматривать как своего рода "фазовый переход". На плоскости $x, t$ при $\varepsilon \rightarrow 0$ выделяются четко разграниченные области: хопфовская область, где решение стремится (в сильном смысле) к решению уравнения Хопфа (3), и уиземовская область, в которой решение испытывает осцилляции конечной амплитуды с длиной волны $\sim \varepsilon$ и имеет предел $\bar{u}(x, t)$ лишь в слабом смысле [12]. Заметим при этом, что сама постановка вопроса о причинах и механизме возникновения осцилляционной области естественна лишь в рамках второго подхода, тогда как применение модуляционных уравнений Уизе- 
ма требует допушения о наличии такой области в качестве дополнительной гипотезы. Прямой анализ при $\varepsilon \rightarrow 0$ можно считать обоснованием этой гипотезы.

Целью настоящей работы является нахождение зависимости модуляционных параметров от $x, t$ в конечном виде, т.е. получение решения уравнений Уизема прямо из явной формулы для $N$-солитонного решения. Использован метод Лакса-Левермора [12], состоящий в редукции исходной задачи к некоторой вариационной задаче с ограничением и решении возникаюшего интегрального уравнения (см. ниже уравнение (11)) путем сведения к проблеме Римана-Гильберта теории функций комплексного переменного. Это позволяет в полной мере использовать результаты работы [12]. Отличие от [12] состоит в том, что решение уравнения (11) строится для правых частей более общего вида, что позволяет получить не только уравнения Уизема, как в [12], но и их решение в замкнутом виде.

\section{2. МНОГОСОЛИТОННОЕ РЕШЕНИЕ ДЛЯ КВАЗИКЛАССИЧЕСКИХ СПЕКТРАЛЬНЫХ ДАННЫХ}

Прежде чем перейти к решению поставленной задачи, надо кратко изложить некоторые сведения, касаюшиеся $N$-солитонного решения при $N \rightarrow \infty$. Приводимые формулы лишь в несушественных деталях отличаются от использованных в работе [12].

Для конкретности начальное условие $u_{0}(x)$ берется в виде простой “потенциальной ямы" $u_{0}(x) \leq 0$ с двумя монотонными "склонами", причем правый склон имеет лишь одну точку перегиба (чтобы не было усложнений с несколькими опрокидываниями). Функцию $u_{0}(x)$ подчиним условиям быстрого убывания на $\pm \infty$, а также условиям нормировки

$$
\min u_{0}=-1, \quad \int_{-\infty}^{\infty}\left(-u_{0}\right)^{1 / 2} d x=\pi .
$$

$N$-солитонное решение, которым при асимптотическом исследовании заменяется решение задачи (1), (2), дается формулой [18]

$$
u(x, t)=-2 \varepsilon^{2} \partial^{2} / \partial x^{2} \ln \sum_{\mu} \exp \left(N^{2} Q_{\mu}\right), \quad Q_{\mu}=-A_{\mu} x+B_{\mu}
$$

где суммирование ведется по всем двоичным словам $\mu=\mu_{1}, \mu_{2}, \ldots, \mu_{N}, \mu_{n}=0$ или 1 , и

$$
\begin{gathered}
A_{\mu}=2 N^{-1} \sum_{n=1}^{N} \mu_{n} k_{n} \\
B_{\mu}=N^{-1} \sum_{n=1}^{N} \mu_{n}\left(8 t k_{n}^{3}+l_{n}\right)+N^{-2} \sum_{m=1}^{N} \sum_{n=1}^{N} \mu_{m}\left(1-\mu_{n}\right) l_{m n} \\
l_{m n}=\ln \left|\left(k_{m}+k_{n}\right) /\left(k_{m}-k_{n}\right)\right| \quad(m \neq n)
\end{gathered}
$$

$\varepsilon$ и $N$ связаны соотношением $N=\varepsilon^{-1}+O(1)$. Параметры $k_{n}, l_{n}$ рассчитьваются по начальному потенциалу $u_{0}(x)$ с помошью квазиклассических формул

$$
k_{n}=k\left(\nu_{n}\right), \quad \nu_{n}=(n-1 / 2) / N, \quad l_{n}=L\left(k_{n}\right)
$$


$k(\nu)$ - обратная функция к

$$
\begin{aligned}
& \nu(k)=\pi^{-1} \int_{\xi_{-}}^{\xi^{+}}\left[-k^{2}-u_{0}(x)\right]^{1 / 2} d x \\
& L(k)=\int_{0}^{k} s\left[\xi_{+}(s)+\xi_{-}(s)\right]\left(k^{2}-s^{2}\right)^{-1 / 2} d s,
\end{aligned}
$$

$\xi_{-}<\xi_{+}-$корни уравнения $u_{0}(\xi)=-k^{2}$. Смысл величины $\nu(k)$ состоит в том, что $N \nu(k)$ есть число собственных значений $\lambda_{n}=-k_{n}^{2}$ дискретного спектра уравнения Шрединге$\mathrm{pa}-\varepsilon^{2} \psi^{\prime \prime}+u_{0}(x) \psi=\lambda \psi$, меньших $-k^{2}$. Уравнение $(7)$ получается при $\varepsilon \rightarrow 0$ из условия квантования Бора-Зоммерфельда.

По виду выражения (4) понятно, что при $N \rightarrow \infty$ основной вклад в сумму по $\mu$ определяется окрестностью максимума величины $Q_{\mu}$. Далее, нормированные указанным в (5), (6) способом суммы преврашаются при $N \rightarrow \infty$ в интегралы, если учесть, что двоичное слово $\mu$ имеет “локальную долю единиц"

$$
p(\nu)=N^{-1} \sum_{n=1}^{N} \mu_{n} \delta\left(\nu-\nu_{n}\right)
$$

которую удобно рассматривать как функцию переменной $k$, связанной с $\nu$ посредством (7). Функция $Q_{\mu}$ на множестве двоичных слов длины $N$ преврашается при $N \rightarrow \infty$ в функционал $Q[p]$ на множестве функций $p(k)$, определенных в интервале $0<k<1$ и изменяюшихся в пределах $0 \leq p(k) \leq 1$. Детальное обоснование этой конструкции дано в [12]. Функционал $Q$ определяется формулой

$$
\begin{aligned}
Q[p]= & \int_{0}^{1}\left[-2 x k+8 t k^{3}+L(k)+h(k)\right] \gamma(k) p(k) d k- \\
& -\int_{0}^{1} \int_{0}^{1} l\left(k, k^{\prime}\right) \gamma(k) \gamma\left(k^{\prime}\right) p(k) p\left(k^{\prime}\right) d k d k^{\prime}
\end{aligned}
$$

где обозначено

$$
\gamma(k)=-d \nu / d k, \quad l\left(k, k^{\prime}\right)=\ln \left|\left(k+k^{\prime}\right) /\left(k-k^{\prime}\right)\right|, \quad h(k)=\int_{0}^{1} l\left(k, k^{\prime}\right) \gamma\left(k^{\prime}\right) d k^{\prime} .
$$

Функция $h(k)$ допускает представление, аналогичное (8):

$$
h(k)=\int_{0}^{k} s\left[\xi_{+}(s)-\xi_{-}(s)\right]\left(k^{2}-s^{2}\right)^{-1 / 2} d s .
$$




\section{3. ЗАДАЧА МАКСИМИЗАЦИИ ФУНКЦИОНАЛА $Q$ ПРИ ДОПОЛНИТЕЛЬНОМ ОГРАНИЧЕНИИ И СООТВЕТСТВУЮШАЯ ВАРИАЦИОННАЯ ЗАДАЧА}

Таким образом, исследование асимптотики решения задачи (1), (2) при $\varepsilon \rightarrow 0$ приводит к проблеме отыскания максимума функционала $Q[p]$ при ограничении $0 \leq p(k) \leq 1$. Обозначим $\hat{p}(k)$ ту функцию $p$, для которой этот максимум достигается.

Область значений $k$, при которых $\hat{p}(k)$ отлично как от 0 , так и от 1 , обозначим $D=$ $D(x, t)$. Для $k$ из этой области функция $p(k)$ имеет возможность в некоторых пределах свободно варьироваться, "не упираясь" в ограничение $0 \leq p(k) \leq 1$. В соответствии с этим замечанием (детальный анализ см. в [12]) для решения рассматриваемой задачи на максимум надо для всех $k \in D$ удовлетворить уравнению

$$
\delta Q[p] / \delta p(k)=0 .
$$

Вне области $D$ либо $\hat{p}(k)=0$, либо $\hat{p}(k)=1$. Учитывая явный вид функционала $Q$, находим, что (10) эквивалентно интегральному уравнению

$$
\int_{D} l\left(k, k^{\prime}\right) \psi\left(k^{\prime}\right) d k^{\prime}=f(k) \quad(\forall k \in D),
$$

где в случае, когда $\hat{p}(k) \equiv 0$ вне $D$,

$$
\psi=\gamma \hat{p}, \quad f=-x k+4 t k^{3}+1 / 2[L(k)+h(k)],
$$

а если $\hat{p}(k) \equiv 1$ вне $D$, то

$$
\psi=\gamma(1-\hat{p}), \quad f=x k-4 t k^{3}-1 / 2[L(k)-h(k)] .
$$

Более сложный случай, когда вне $D$ бывает и $\hat{p}(k)=0$, и $\hat{p}(k)=1$, рассмотрен в разделе 5 .

Особенностью рассматриваемой задачи является то, что область $D$ заранее не известна. При заданной же области $D$ в том случае, если она состоит из конечного числа интервалов, для решения уравнения (11) можно использовать [12] известную краевую задачу Римана-Гильберта теории функций комплексного переменного; ниже это подробно изложено. При заранее неизвестной области $D$ приходится исходить из некоторых априорных представлений об ее устройстве, которые проверяются после полного решения задачи и сводятся к следуюшему.

Для $t$, не превышающих некоторого значения $t_{*}$ (которое, как ясно из дальнейшего, есть как раз момент "опрокидывания" решения уравнения (3)), область $D$ представляет собой один интервал

$$
D=(0, c) .
$$

При этом имеется такое $x_{1}=x_{1}(t)$, что при $x<x_{1} \hat{p}(k) \equiv 1$ для $k$ вне $D$, а при $x>x_{1}$ вне $D$ тождественно $\hat{p}(k)=0$. Эта ситуация представлена на рис. $1 a$, где изображены 

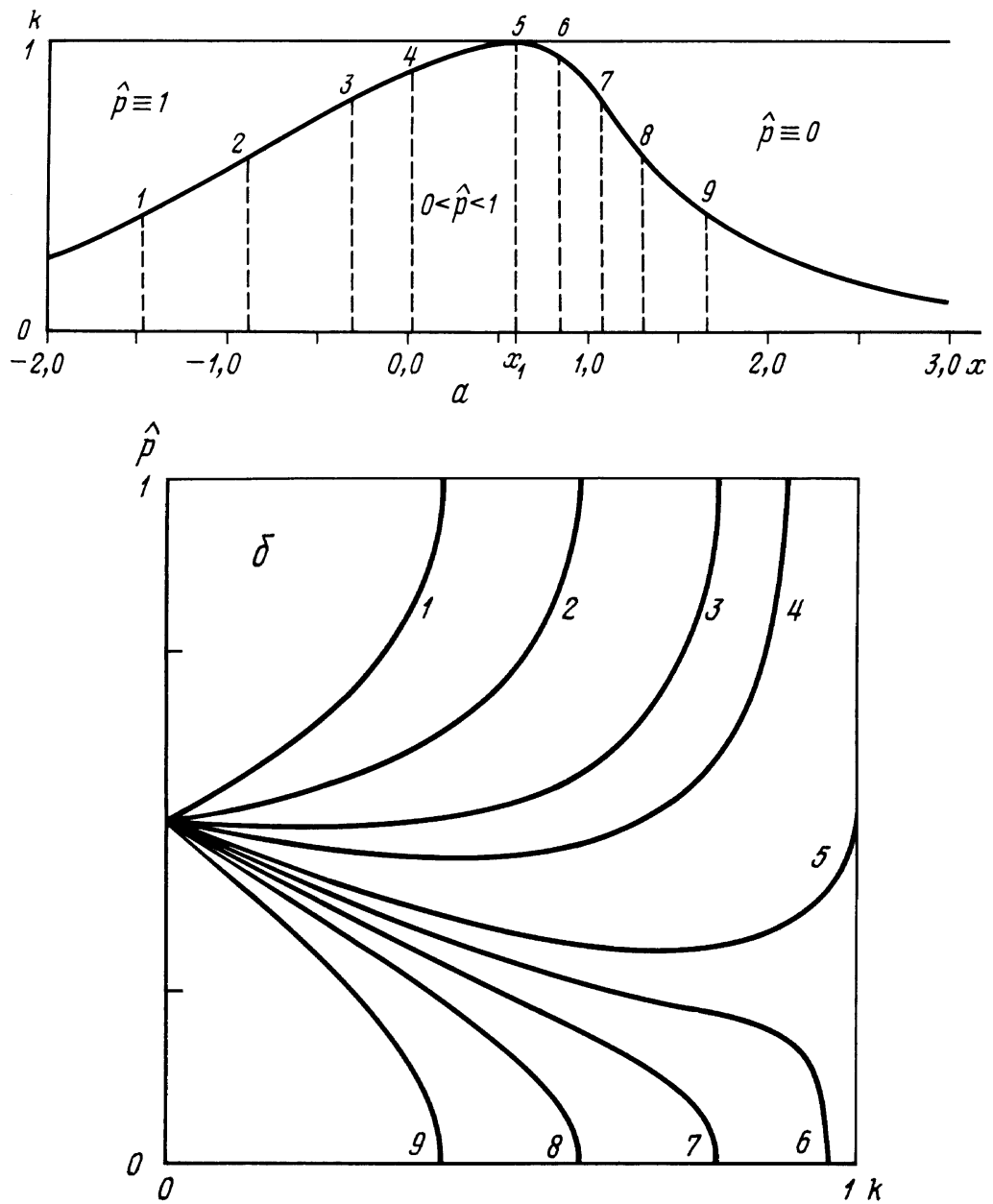

Рис. 1

область определения функции $\hat{p}(k ; x)$ (полоса $0 \leq k \leq 1$ ) и график функции $k=c(x)$, разделяюший ее на части, где соответственно $\hat{p} \equiv 1,0<\hat{p}<1, \hat{p} \equiv 0$. На рис. 16 для того же момента времени $t<t_{*}$ показаны графики максимизатора $\hat{p}(k)$ при некоторых значениях $x$ (отмеченных на рис.1 $a$ ). При $t>t_{*}$ возникает интервал значений $x: x^{-}(t)<x<x^{+}(t)$, для которых область $D(x, t)$ разделяется на два интервала (см.рис. $2 a, 6)$ :

$$
D=(0, a) \cup(b, c),
$$

причем $\hat{p}(k) \equiv 0$ вне $D$. Для значений $x \notin\left(x^{-}, x^{+}\right)$по-прежнему $D$ состоит из одного интервала. С дальнейшим ростом $t$ происходит некоторое усложнение ситуации после момента $t_{1}$, когда точка $x_{1}(t)$ оказывается правее точки $x^{-}(t)$. При $t>t_{1}$ функция $\hat{p}(k)$ вне $D$ перестает быть тождественным нулем, интервал $\left(x^{-}, x^{+}\right)$разбивается точкой $x_{1}$ 

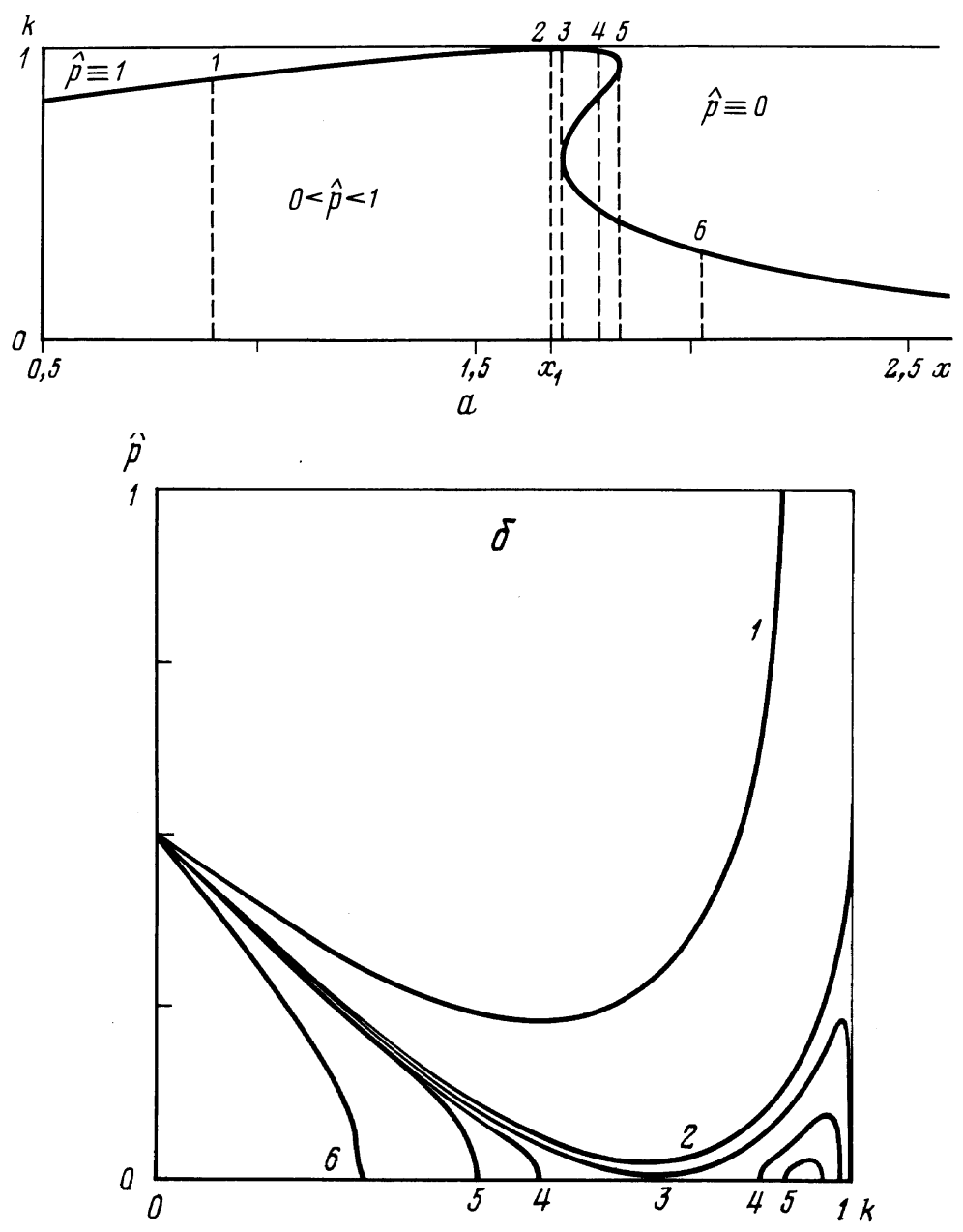

Рис. 2

на две части, и для $x \in\left(x^{-}, x_{1}\right)$ во внешности $D(x, t)$ мы имеем

$$
\hat{p}(k)=0 \text { при } a \leq k \leq b, \quad \hat{p}(k)=1 \text { при } k \geq c,
$$

что иллюстрируется относящимися к случаю $t>t_{1}$ рисунками $3 a$ и 36 .

Приведенные на рисунках графики соответствуют начальному условию $u_{0}(x)=$ $-(\operatorname{ch} x)^{-2}$, для которого $L(k)=0, \gamma(k)=1$ (т.е. либо $\hat{p}=\psi$, либо $\hat{p}=1-\psi$ ). Решение уравнения $(11)$ в этом частном случае для $D=(0, c)$ может быть получено методом задачи Римана-Гильберта (как описано ниже в разделе 4) и выражается в элементарных функциях:

$$
\psi=\pi^{-1}\left(\operatorname{arctg} \frac{\sqrt{c^{2}-k^{2}}}{k \sqrt{1-c^{2}}} \mp 12 t k \sqrt{c^{2}-k^{2}}\right)
$$



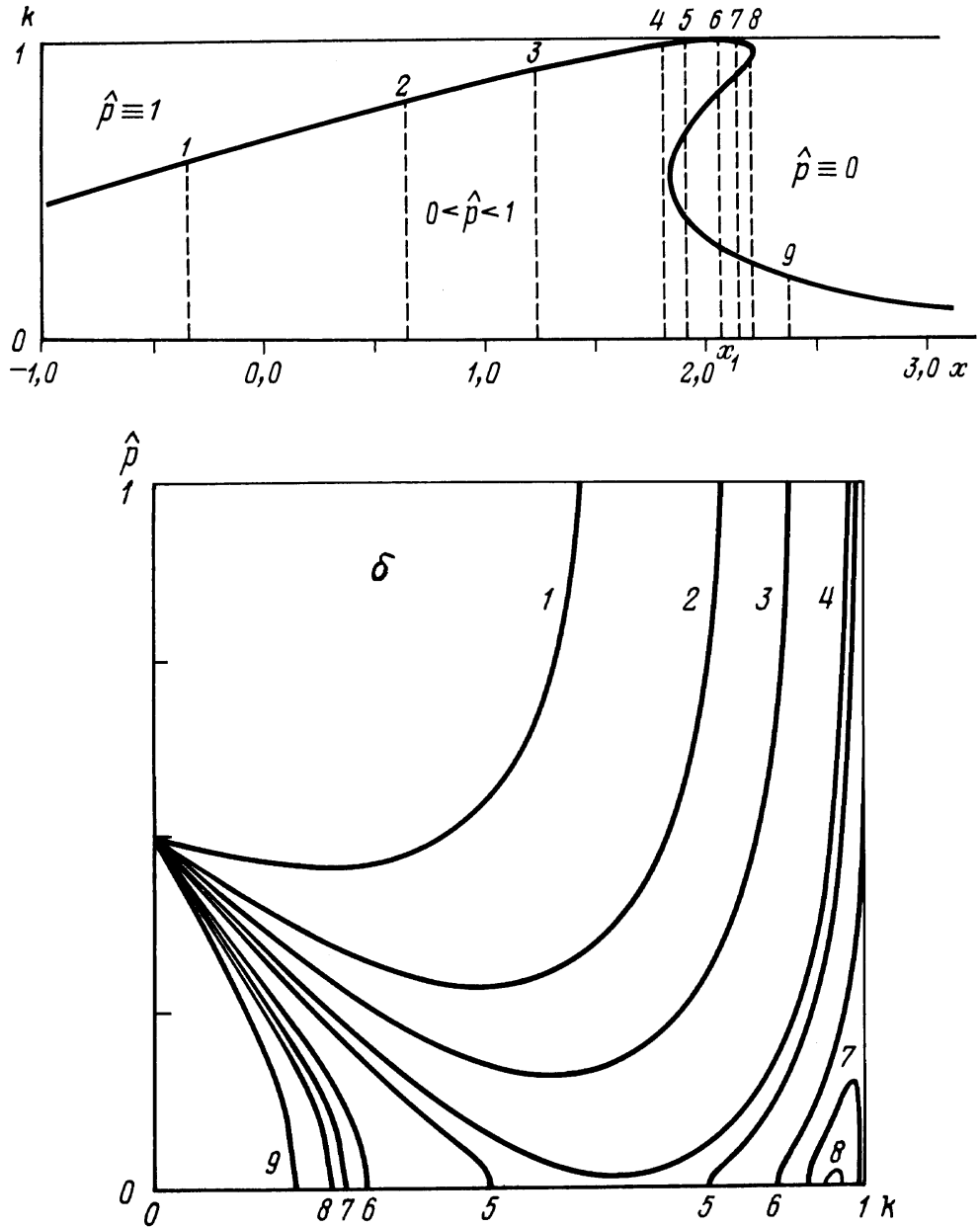

Рис. 3

(верхний знак относится к случаю, когда $\hat{p}=\psi$, нижний - к случаю, когда $\hat{p}=1-\psi$ ). Все кривые на рис. 16 , а также кривые $1-3,6$ на рис. 26 и 1-4, 9 на рис. 36 рассчитаны по указанной формуле. При $t>t_{*}$, когда $D=(0, a) \cup(b, c)$, элементарного решения, разумеется, не получается; соответствующие кривые $(4,5$ на рис. 26 и 5-8 на рис. 36$)$ имеют качественный характер.

\section{4. РЕШЕНИЕ ВАРИАЦИОННОЙ ЗАДАЧИ И НАХОЖ ДЕНИЕ ЗАВИСИМОСТИ МОДУЛЯЦИОННЫХ ПАРАМЕТРОВ ОТ $x, t$ ПРИ $t \leq t_{1}$}

Ограничиваясь вначале случаем $t \leq t_{1}$, мы приходим к необходимости решить уравнение (11) для $D$ вида (12) при $t \leq t_{*}$ или вида (13) для некоторых $x$ при $t>t_{*}$.

Решение уравнения (11) сводится к задаче Римана-Гильберта. Этот метод был ис- 
пользован в [12] для нахождения $\partial \psi / \partial x$ и $\partial \psi / \partial t$ из продифференцированного соответственно по $x$ и $t$ уравнения (11). Правая часть продифференцированного уравнения представляет собой просто нечетную степень $k$, и решение задачи Римана-Гильберта получается чисто алгебраически. Найденные выражения для $\partial \psi / \partial x$ и $\partial \psi / \partial t$ позволили получить (при $t>t_{*}$ ) для величин $a^{2}, b^{2}, c^{2}$ как функций $x, t$ систему дифференциальных уравнений, которая оказалась не чем иным, как системой уравнений Уизема в римановой форме:

$$
\partial_{t} r_{i}+v_{i}\left(r_{1}, r_{2}, r_{3}\right) \partial_{x} r_{i}=0, \quad i=1,2,3
$$

где

$$
r_{1}=a^{2}, \quad r_{2}=b^{2}, \quad r_{3}=c^{2} .
$$

Выражения для $v_{i}$ приведены ниже (формулы $(29)$ ).

Этот факт является одним из основных результатов серии работ [12]. Отметим, что в [12] получены более обшие уравнения - для границ любого числа интервалов, составляющих область $D$.

В настояшей работе использован тот же метод сведения уравнения (11) к задаче Римана-Гильберта, но для нахождения самой функции $\psi(k)$, а не ее производных по $x$ и $t$. При этом для величин $a^{2}, b^{2}, c^{2}$ получается система конечных уравнений, которая дает в неявной форме решение уравнений Уизема. Это и естественно, поскольку при дифференцировании уравнения (11) по $x$ или $t$ пропадает член $\pm L+h$, содержаший информацию о начальных данных, тогда как при решении самого уравнения (11) эта информация сохраняется. Поясним, каким образом получается эта система конечных соотношений, на примере $D=(0, a)(b, c)$. Функция $\psi(k)$, определяемая из уравнения $(11)$, при произвольном расположении точек $a, b, c$, вообще говоря, неограниченна в окрестности этих точек. Она ведет себя как

$$
C_{\xi}(a, b, c) \rho^{-1 / 2}, \quad \xi=a, b, c, \quad \rho=|k-\xi|
$$

Вместе с тем основное условие $0 \leq \hat{p} \leq 1$ требует ограниченности функции $\psi$. Поэтому три коэффициента $C_{\xi}(a, b, c)$, зависящие также (очевидно, линейно) от параметров $x, t$ уравнения (11), должны быть равны нулю. Получается как раз система трех уравнений относительно трех неизвестных $a, b, c$, неявно определяющая их как функции от $x, t$.

Задача Римана-Гильберта состоит в том, чтобы найти аналитическую в верхней полуплоскости функцию комплексного переменного $\Phi(z), z=k+i k^{\prime}$, по заданным на чередующихся интервалах действительной оси ее мнимой и действительной частям $\operatorname{Im} \Phi(k+i 0), \operatorname{Re} \Phi(k+i 0)$. Чтобы связать уравнение $(11)$ с этой задачей, напишем его в более симметричной форме, доопределив при $k>0$ искомую функцию $\psi(k)$ нулем вне области $D$ и затем продолжив ее нечетным образом для $k \leq 0$. Вследствие нечетности $l\left(k, k^{\prime}\right)$ по каждому из аргументов уравнение (11) заменяется после дифференцирования по $k$ уравнением [12]

$$
f_{-\infty}^{\infty} \frac{\psi\left(k^{\prime}\right)}{k^{\prime}-k} d k^{\prime}=f^{\prime}(k) \quad(\forall k \in \Delta),
$$

где $f(k)$ - нечетное продолжение правой части (11), а область $\Delta$ состоит из $D$, отражения $D$ относительно нуля и точки $k=0$. 
Обратим внимание, что (16) есть следствие (11), но не наоборот. Если область $D$ несвязна, как, например, в случае (13), то (11) нельзя получить из (16) просто интегрированием от 0 до $k$, поскольку в интервале $(a, b)$ соотношение (16) не обязано выполняться. Поэтому при наличии “дырок" в области $D$ надо накладьвать на $\psi(k)$ дополнительные условия, состоящие в том, что интеграл от левой части (16) по каждому промежутку, выпадающему из $D$, равен приращению функции $f(k)$ на этом промежутке (см. ниже условие (20)).

Сопоставим искомой функции $\psi(k)$ аналитическую в верхней полуплоскости функцию $\Phi(z)$, у которой $\operatorname{Im} \Phi(k+i 0)=\psi(k)$. Эта функция определяется с точностью до действительной константы, равной $\Phi(\infty)$. Положим для определенности $\Phi(\infty)=0$, тогда

$$
\operatorname{Re} \Phi(k+i 0)=\pi^{-1} f_{-\infty}^{\infty} \frac{\psi\left(k^{\prime}\right)}{k^{\prime}-k} d k^{\prime}
$$

Сопоставляя с (16), мы видим, что функция $\Phi(z)$ (зная которую, можно найти искомую $\psi(k)=\operatorname{Im} \Phi(k+i 0))$ удовлетворяет условиям

$$
\begin{aligned}
& \operatorname{Re} \Phi(k+i 0)=\pi^{-1} f^{\prime}(k), \quad \forall k \in \Delta, \\
& \operatorname{Im} \Phi(k+i 0)=0, \quad \forall k \notin \Delta,
\end{aligned}
$$

т.е. условиям задачи Римана-Гильберта и, кроме того, условию $\Phi(\infty)=0$.

Решение задачи Римана-Гильберта с граничным условием (17) не является единственным. Одно из решений дается формулой Келдыша-Седова [19], которую в данном случае удобно использовать в модифицированном виде

$$
\Phi_{0}(z)=\left(i \pi^{2}\right)^{-1} r(z) \int_{\Delta} f^{\prime}(\varkappa) r(\varkappa)^{-1}(\varkappa-z)^{-1} d \varkappa,
$$

где $r(z)=[P(z)]^{1 / 2} ; P(z)=z^{2}-c^{2}, \Delta=(-c, c)$ в случае (12) и $P(z)=\left(z^{2}-a^{2}\right) \times$ $\left(z^{2}-b^{2}\right)\left(z^{2}-c^{2}\right), \Delta=(-c,-b) \cup(-a, a) \cup(b, c)$ в случае (13). Функция $r(z)$ рассматривается в верхней полуплоскости, причем берется ее ветвь, положительная в точках действительной оси, расположенных правее области $\Delta$.

Добавление к $\Phi_{0}(z)$ функции вида $Q(z) / r(z)$, где $Q(z)$ - любая функция, аналитическая в верхней полуплоскости и действительная на действительной оси, очевидно, не нарушает условия (17). Поэтому будем искать нужное для получения решения уравнения (11) решение задачи Римана-Гильберта в виде

$$
\Phi=\Phi_{0}+Q / r
$$

При этом достаточно рассматривать в качестве $Q(z)$ полиномы с вешественными коэффициентами.

Искомое решение выделяется условиями $\Phi(\infty)=0$ и нечетности $\operatorname{Im} \Phi(k+i 0)$. В случае (13) добавляется еше одно условие, вызванное несвязностью области $D$ : чтобы при 
интегрировании (16) получить (11), надо потребовать соблюдения дополнительного условия

$$
\int_{a}^{b}\left[\operatorname{Re} \Phi(k+i 0)-\pi^{-1} f^{\prime}(k)\right] d k=0 .
$$

Степень полинома $Q(z)$ определяется поведением $\Phi_{0}(z)$ при $z \rightarrow \infty$ : если $\Phi_{0} \sim z^{n}$, то степень полинома $Q$ равна $n+\nu, \nu=1$ в случае (12) и $\nu=3$ в случае (13).

условие нечетности $\operatorname{Im} \Phi(k+i 0)$ учитывается очень просто благодаря тому, что $\operatorname{Im} \Phi_{0}(k+i 0)$ обладает этим свойством. У полинома $Q(z)$ поэтому отличны от нуля лишь коэффишиенты при нечетных степенях $z$.

Благодаря линейности уравнения (11) можно решить задачу (17) отдельно для $f=k$, $f=k^{3}$ и $f=f^{( \pm)}(k)=(L \pm h) / 2$, удовлетворить указанным выше дополнительным условиям, а затем составить из решений нужную линейную комбинацию. При этом для функций $f=k, k^{3}$ частное решение $\Phi_{0}$ указывается тривиально: очевидно, $\Phi_{0}^{(1)}(z)=\pi^{-1}$ и $\Phi_{0}^{(3)}(z)=3 \pi^{-1} z^{2}$ удовлетворяют соответствуюшим условиям (17). Такого рода решения, дополненные $Q / r$, использованы в [12]. Для функции же $f^{( \pm)}$приходится реально применять формулу Келдьша-Седова (18), причем полезно следуюшее вытекаюшее из $(8),(9)$ представление через начальный потеншиал $u_{0}(x)$ :

$$
f^{( \pm)}(k)=\int_{0}^{k} s \xi_{ \pm}(s)\left(k^{2}-s^{2}\right)^{-1 / 2} d s
$$

(преобразование Абеля), где $\xi_{ \pm}(s)$ - корни уравнения $u_{0}(\xi)=-s^{2}$.

Теперь рассмотрим последовательно случаи (12) и (13).

Случай $\boldsymbol{D}=(\mathbf{0}, \boldsymbol{c})$. Для степенных функций $k, k^{3}$, определяя коэффициенты полиномов $Q(z)$ из условия $\Phi(\infty)=0$, находим

$$
\begin{aligned}
& \Phi^{(1)}(z)=\pi^{-1}\left[1-z\left(z^{2}-c^{2}\right)^{-1 / 2}\right] \\
& \Phi^{(3)}(z)=3 \pi^{-1}\left[z^{2}-\left(z^{3}-2^{-1} c^{2} z\right)\left(z^{2}-c^{2}\right)^{-1 / 2}\right] .
\end{aligned}
$$

Для $f=f^{( \pm)}$после подстановки (21) в (18), изменения порядка интегрирований и явного вычисления внутреннего интеграла с помощью вычетов получаем

$$
\Phi_{0}^{( \pm)}(z)=z \pi^{-1}\left[\left(z^{2}-c^{2}\right)^{-1 / 2} \xi_{ \pm}(c)-\int_{0}^{c}\left(z^{2}-s^{2}\right)^{-3 / 2} s \xi_{ \pm}(s) d s\right] .
$$

Условие $\Phi^{( \pm)}(\infty)=0$ (с учетом поведения функции $(22)$ при $z \rightarrow \infty \quad \Phi_{0}^{( \pm)}(z)=$ $\left.\pi^{-1} \xi_{ \pm}(c)+O\left(z^{-2}\right)\right)$ определяет полином $Q(z)$, в данном случае - первой степени. Имеем

$$
\Phi^{( \pm)}(z)=\Phi_{0}^{( \pm)}(z)-\pi^{-1} \xi_{ \pm}(c) z\left(z^{2}-c^{2}\right)^{-1 / 2} .
$$

Теперь можно составить нужную линейную комбинацию функций $\Phi^{(1)}, \Phi^{(3)}, \Phi^{( \pm)}$:

$$
\Phi(z)=\Phi_{0}(z) \pm \pi^{-1} z\left(z^{2}-c^{2}\right)^{-1 / 2}\left[x+12 t\left(c^{2} / 2-z^{2}\right)-\xi_{ \pm}(c)\right],
$$


где $\Phi_{0}$ - соответствуюшая комбинация частных решений $\Phi_{0}^{(1)}, \Phi_{0}^{(3)}, \Phi_{0}^{( \pm)}$. Отсюда видно, что для ограниченности $\operatorname{Im} \Phi(k+i 0)$ при $k \rightarrow c-0$ (учитывая, что $\operatorname{Im} \Phi_{0}(k+i 0)$ ограничена) надо связать $c, x, t$ соотношением $x=6 t c^{2}+\xi_{ \pm}(c)$. Это означает, что зависимость границы области $D$ от $x, t$ определяется решением уравнения Хопфа $(3) \mathrm{c}$ начальным условием $u(x, 0)=u_{0}(x)$ :

$$
c^{2}=-u(x, t) .
$$

Случай $\boldsymbol{D}=(\mathbf{0}, \boldsymbol{a}) \cup(\boldsymbol{b}, \boldsymbol{c})$. Полиномы $Q^{(n)}$ в решениях для $f=k, k^{3}$

$$
\Phi^{(1)}(z)=\pi^{-1}+Q^{(1)}(z) / r(z), \quad \Phi^{(3)}(z)=3 \pi^{-1} z^{2}+Q^{(3)}(z) / r(z)
$$

определяются с помощью условий $\Phi^{(n)}(\infty)=0$ и $(20)$ :

$$
\begin{aligned}
& Q^{(1)}(z)=z \pi^{-1} J_{1}^{-1}\left(J_{3}-z^{2} J_{1}\right), \\
& Q^{(3)}(z)=3 z \pi^{-1} J_{1}^{-1}\left[J_{5}-z^{4} J_{1}-\frac{1}{2} \sigma\left(J_{3}-z^{2} J_{1}\right)\right],
\end{aligned}
$$

где обозначено $\sigma=a^{2}+b^{2}+c^{2}$ и

$$
J_{n}=\int_{a}^{b} k^{n}[P(k)]^{-1 / 2} d k .
$$

Функции $J_{n}(a, b, c)$ выражаются через стандартные полные эллиптические интегралы $\mathrm{K}(m), \mathrm{E}(m)$; эти формулы приведены в приложении.

Решение (18) для $f=f^{(+)}(k)$ с помошью процедуры, аналогичной выводу формулы (22), приводится к виду

$$
\begin{gathered}
\Phi_{0}^{(+)}(z)=\Phi_{0}^{(+)(0, c)}(z)+\Psi(z), \\
\Psi(z)=-\pi^{-2} z r(z)\left[\int_{a}^{b} \xi_{+}^{\prime}(s) d s \int_{a^{2}}^{s^{2}} G d \zeta+\int_{b}^{c} \xi_{+}^{\prime}(s) d s \int_{a^{2}}^{b^{2}} G d \zeta\right],
\end{gathered}
$$

где $G=\left(\zeta-z^{2}\right)^{-1}\left[P\left(\zeta^{1 / 2}\right)\left(s^{2}-\zeta\right)\right]^{-1 / 2}$, а $\Phi_{0}^{(+)(0, c)}$ обозначает здесь и ниже частное решение задачи Римана-Гильберта для случая $D=(0, c)$ и $f=f^{(+)}$, которое определяется выражением (22). Выделение слагаемого $\Phi_{0}^{(+)(0, c)}$ в $(25)$ облегчает применение условия $(20)$. Далее, зная $\Phi_{0}^{(+)}(z)$, можно найти полином $Q^{(+)}(z)$ в $\Phi^{(+)}=\Phi_{0}^{(+)}+Q^{(+)} / r$. При этом степень полинома и его коэффициенты, кроме одного, определяются условием $\Phi^{(+)}(\infty)=0$ с учетом поведения $\Phi_{0}^{(+)}(z)$ при $z \rightarrow \infty$ :

$$
\begin{gathered}
Q^{(+)}(z)=\pi^{-1}\left(A_{5} z^{5}+A_{3} z^{3}+A_{1} z\right) \\
A_{5}=\pi^{-1} \int_{a}^{c} \Gamma_{0}(s) \xi_{+}^{\prime}(s) d s \\
A_{3}=-\xi_{+}(c)+\pi^{-1} \int_{a}^{c}\left[\sigma \Gamma_{0}(s)-\Gamma_{1}(s)\right] \xi_{+}^{\prime}(s) d s
\end{gathered}
$$


где

$$
\Gamma_{n}(s)=\int_{a^{2}}^{\mu} \zeta^{n}\left[P\left(\zeta^{1 / 2}\right)\left(s^{2}-\zeta\right)\right]^{-1 / 2} d \zeta, \quad \mu=\min \left(s^{2}, b^{2}\right) .
$$

Функции $\Gamma_{n}(s)$ выражаются через полные эллиптические интегралы первого и третьего родов (см. приложение). Оставшийся коэффициент $A_{1}$ находится из условия (20), которое, с учетом того что для $k \in(a, b)$ известна $\operatorname{Re} \Phi_{0}^{(+)(0, c)}(k+i 0)=\pi^{-1} f^{\prime}(k)$, принимает вид

$$
A_{5} J_{5}+A_{3} J_{3}+A_{1} J_{1}=\pi \int_{a}^{b} \operatorname{Re} \Psi(k+i 0) d k .
$$

Правую часть этого соотношения обозначим $B$; как и коэффициенты $A_{5}, A_{3}$, она приводится к виду линейного функционала от $\xi_{+}(s)$ :

$$
B=\pi^{-1} \int_{a}^{c} \beta(s) \xi_{+}^{\prime}(s) d s
$$

с ядром

$$
\beta(s)=\int_{a}^{b} k[P(k)]^{1 / 2} d k f_{a^{2}}^{\mu}\left(\zeta-k^{2}\right)^{-1}\left[P\left(\zeta^{1 / 2}\right)\left(s^{2}-\zeta\right)\right]^{-1 / 2} d \zeta .
$$

Выражение $\beta(s)$ через эллиптические интегралы дано в приложении. Полином $Q^{(+)}$ полезно представить в виде

$$
Q^{(+)}(z)=z \pi^{-1} J_{1}^{-1}\left[B-\left(J_{5}-z^{4} J_{1}\right) A_{5}-\left(J_{3}-z^{2} J_{1}\right) A_{3}\right] .
$$

Теперь можно, как и в случае $D=(0, c)$, составить из $(23),(24)$ и $(28)$ нужную линейную комбинацию $Q(z)$. Вследствие ограниченности $\operatorname{Im} \Phi_{0}(k+i 0)$ условиями ограниченности $\operatorname{Im} \Phi(k+i 0)$ при $k \rightarrow a, b, c$ являются равенства $Q(a)=0, Q(b)=0, Q(c)=0$, т.е. в явном виде

$$
\begin{aligned}
& -x\left(J_{3}-z^{2} J_{1}\right)+12 t\left[J_{5}-z^{4} J_{1}-\frac{1}{2} \sigma\left(J_{3}-z^{2} J_{1}\right)\right]+ \\
& \quad+B-\left(J_{5}-z^{4} J_{1}\right) A_{5}-\left(J_{3}-z^{2} J_{1}\right) A_{3}=0, \quad z=a, b, c .
\end{aligned}
$$

После переобозначения $a^{2}=r_{1}, b^{2}=r_{2}, c^{2}=r_{3}$ (cp.(14), (15)) получается система уравнений Царева [5,6]

$$
x=t v_{i}\left(r_{1}, r_{2}, r_{3}\right)+w_{i}\left(r_{1}, r_{2}, r_{3}\right), \quad i=1,2,3 .
$$

Коэффициенты при $t$

$$
v_{i}=12\left(J_{5}-r_{i}^{2} J_{1}\right)\left(J_{3}-r_{i} J_{1}\right)^{-1}-6 \sigma
$$

являются характеристическими скоростями системы уравнений (14). В приложении приведены их выражения через К, Е.

Выражение

$$
w_{i}=-A_{3}-\rho_{i} A_{5}+\left(J_{3}-r_{i} J_{1}\right)^{-1} B, \quad \rho_{i}=\left(J_{5}-r_{i}^{2} J_{1}\right)\left(J_{3}-r_{i} J_{1}\right)^{-1},
$$


необходимо сравнить с результатами работ, посвяшенных решению системы уравнений (14). Важным свойством функций $w_{i}$ является возможность потенциального представления [7]

$$
w_{i}=f-\left(\lambda / \partial_{i} \lambda\right) \partial_{i} f
$$

где $f=f\left(r_{1}, r_{2}, r_{3}\right), \partial_{i} \equiv \partial / \partial r_{i}, \lambda=\mathrm{const} \cdot J_{1}$ - медленно меняюшаяся длина волны в модулированном пакете.

Чтобы представить (30) в виде (31), заметим, что имеют место соотношения

$$
\rho_{i}=\frac{2}{3} \sigma-\frac{1}{6} \lambda / \partial_{i} \lambda, \quad\left(J_{3}-r_{i} J_{1}\right)^{-1}=\alpha_{i} \lambda / \partial_{i} \lambda
$$

где

$$
\begin{gathered}
\sigma=r_{1}+r_{2}+r_{3}, \quad \alpha_{i}=\varkappa_{i}^{-1}\left(r_{3}-r_{1}\right)^{-3 / 2}[2 \mathrm{~K}(m)]^{-1}, \\
\varkappa_{1}=m, \quad \varkappa_{2}=m(m-1), \quad \varkappa_{3}=1-m, \quad m=\left(r_{2}-r_{1}\right) /\left(r_{3}-r_{1}\right) .
\end{gathered}
$$

Выражение (30) преобразуется к виду

$$
w_{i}=-A_{3}-\frac{2}{3} \sigma A_{5}+\left(\lambda / \partial_{i} \lambda\right)\left(\frac{1}{6} A_{5}+\alpha_{i} B\right) .
$$

Эта формула имеет вид представления (31), однако

$$
\partial_{i}\left(A_{3}+\frac{2}{3} \sigma A_{5}\right) \neq \frac{1}{6} A_{5}+\alpha_{i} B
$$

Оказывается, что для получения потенциального представления надо еше перераспределить слагаемые в (32):

$$
w_{i}=-A_{3}-\frac{2}{3} \sigma A_{5}+X-\left(\lambda / \partial_{i} \lambda\right)\left[-\frac{1}{6} A_{5}-\alpha_{i} B+\left(\partial_{i} \lambda / \lambda\right) X\right],
$$

где

$$
X=\pi^{-1} \int_{a}^{c}\left(\frac{1}{3} \sigma-s^{2}\right) \Gamma_{0}(s) \xi_{+}^{\prime}(s) d s .
$$

Таким образом, полученное в данной работе выражение (30) представлено в виде (31) с потенциалом

$$
f=\xi_{+}(c)+\pi^{-1} \int_{a}^{c}\left[-s^{2} \Gamma_{0}(s)+\Gamma_{1}(s)\right] \xi_{+}^{\prime}(s) d s .
$$

В работах $[9,10]$ выражение для $f$ содержит под интегралом $\xi_{+}(s)$, а не $\xi_{+}^{\prime}(s)$. Для сравнения преобразуем (33) с помощью интегрирования по частям; при этом исчезает и внеинтегральный член $\xi_{+}(c)$, и эллиптический интеграл третьего рода $\Gamma_{1}$ (поскольку $\left.\partial_{s} \Gamma_{1}=s^{2} \partial_{s} \Gamma_{0}+s \Gamma_{0}\right)$. Имеем

$$
f=\pi^{-1} \int_{a}^{c} s \Gamma_{0}(s) \xi_{+}(s) d s
$$


Эта формула после замены $y=s^{2}$ в точности совпадает с результатом работы [9] (фор-

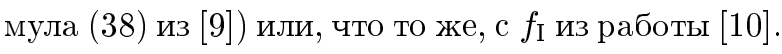

\section{5. АНАЛИЗ СЛУЧАЯ $t>t_{1}$}

В этом случае для $x$ из промежутка $x^{-}(t)<x<x_{1}(t)$ ситуация описывается уравнением $(11)$ с $D=(0, a) \cup(b, c)$ и правой частью

$$
f=-x k+4 t k^{3}+f^{(+)}(k)-\int_{c}^{1} l\left(k, k^{\prime}\right) \gamma\left(k^{\prime}\right) d k^{\prime}
$$

По сравнению с предыдущим рассмотрением появилась добавка в виде интеграла по области $(c, 1)$, где $\hat{p}(k) \equiv 1$. Решения уравнения $(11)$ с правыми частями $k, k^{3}$ и $f^{(+)}(k)$, изученные в разделе 4 , обозначим $\psi^{(1)}, \psi^{(3)}$ и $\psi^{(+)}$. Для решения уравнения (11) с правой частью (35) остается найти при $k_{0}>c$ решение интегрального уравнения

$$
\int_{D} l\left(k, k^{\prime}\right) \psi^{(l)}\left(k^{\prime} ; k_{0}\right) d k^{\prime}=l\left(k, k_{0}\right) \quad(\forall k \in D),
$$

после чего искомое решение получается в виде линейной комбинации

$$
\psi(k)=-x \psi^{(1)}(k)+4 t \psi^{(3)}(k)+\psi^{(+)}(k)-\int_{c}^{1} \psi^{(l)}(k ; \varkappa) \gamma(\varkappa) d \varkappa .
$$

Применяя для решения (36) метод задачи Римана-Гильберта, находим по формуле Келдыша-Седова

$$
\Phi_{0}^{(l)}\left(z ; k_{0}\right)=\frac{2}{\pi r\left(k_{0}\right)} \frac{z r(z)-k_{0} r\left(k_{0}\right)}{z^{2}-k_{0}^{2}} \quad\left(k_{0}>c\right) .
$$

Функцию комплексной переменной $z$, даюшую $\psi^{(l)}$ как мнимую часть при $z \rightarrow k+i 0$, получаем по формуле

$$
\Phi^{(l)}\left(z ; k_{0}\right)=\Phi_{0}^{(l)}\left(z ; k_{0}\right)+Q^{(l)}\left(z ; k_{0}\right) / r(z),
$$

где полином пятой степени $Q^{(l)}$ определяется с учетом дополнительных условий $\Phi^{(l)}\left(\infty ; k_{0}\right)=0$ и $(20)$ :

$$
\begin{gathered}
Q^{(l)}\left(z ; k_{0}\right)=z \pi^{-1} J_{1}^{-1} r\left(k_{0}\right)^{-1}\left[Y\left(k_{0}\right)+2\left(J_{5}-z^{4} J_{1}\right)-2\left(\sigma-k_{0}^{2}\right)\left(J_{3}-z^{2} J_{1}\right)\right], \\
Y\left(k_{0}\right)=\int_{a}^{b} 2 k[P(k)]^{1 / 2}\left(k^{2}-k_{0}^{2}\right)^{-1} d k \quad\left(\sigma=a^{2}+b^{2}+c^{2}\right) .
\end{gathered}
$$

Наконец, находим функцию

$$
\Phi(z)=-x \Phi^{(1)}(z)+4 t \Phi^{(3)}(z)+\Phi^{(+)}(z)-\int_{c}^{1} \Phi^{(l)}(z ; \varkappa) \gamma(\varkappa) d \varkappa
$$


даюшую $(37)$ как $\psi(k)=\operatorname{Im} \Phi(k+i 0)$.

Соответственно полином $Q(z)$ в представлении $\Phi=\Phi_{0}+Q / r$ имеет вид

$$
Q=-x Q^{(1)}+4 t Q^{(3)}+Q^{(+)}+Q^{*}
$$

где $Q^{(1)}, Q^{(3)}, Q^{(+)}$- полиномы, найденные в разделе 4 (формулы $(23),(24)$ и $\left.(28)\right)$, а полином

$$
Q^{*}(z)=-\int_{c}^{1} Q^{(l)}(z ; \varkappa) \gamma(\varkappa) d \varkappa
$$

представляется в виде, аналогичном (28):

$$
Q^{*}(z)=z \pi^{-1} J_{1}^{-1}\left[B^{*}-\left(J_{5}-z^{4} J_{1}\right) A_{5}^{*}-\left(J_{3}-z^{2} J_{1}\right) A_{3}^{*}\right]
$$

где выражениям для $B^{*}, A_{5}^{*}, A_{3}^{*}$ можно придать форму, аналогичную формулам для $B$, $A_{5}, A_{3}$ из раздела 4 . Воспользуемся выражением для $\gamma=d \nu / d k$ :

$$
\gamma(k)=-k \pi^{-1} \int_{k}^{1}\left(s^{2}-k^{2}\right)^{-1 / 2} \Delta^{\prime}(s) d s, \quad \Delta=\xi_{+}-\xi_{-} .
$$

После подстановки (39) в (38) и изменения порядка интегрирований получаются выражения

$$
\left(B^{*}, A_{5}^{*}, A_{3}^{*}\right)=\pi^{-1} \int_{c}^{1}\left(\beta^{*}(s),-\Gamma_{0}^{*}(s), \sigma \Gamma_{0}^{*}(s)-\Gamma_{1}^{*}(s)\right) \Delta^{\prime}(s) d s,
$$

где

$$
\begin{aligned}
& \Gamma_{n}^{*}(s)=\int_{c^{2}}^{s^{2}} \zeta^{n}\left[P\left(\zeta^{1 / 2}\right)\left(s^{2}-\zeta\right)\right]^{-1 / 2} d \zeta, \quad n=0,1, \\
& \beta^{*}(s)=\int_{a}^{b} k[P(k)]^{1 / 2} d k \int_{c^{2}}^{s^{2}}\left(k^{2}-\zeta\right)^{-1}\left[P\left(\zeta^{1 / 2}\right)\left(s^{2}-\zeta\right)\right]^{-1 / 2} d \zeta
\end{aligned}
$$

Условия ограниченности $\psi(k)$ в точках $a, b, c$ имеют после переобозначений $a^{2}=r_{1}$, $b^{2}=r_{2}, c^{2}=r_{3}$ вид

$$
x=t v_{i}\left(r_{1}, r_{2}, r_{3}\right)+W_{i}\left(r_{1}, r_{2}, r_{3}\right), \quad i=1,2,3,
$$

где $W_{i}=w_{i}+w_{i}^{*} ; v_{i}, w_{i}$ найдены в разделе 4 (формулы $\left.(29),(30)\right)$, а

$$
w_{i}^{*}=\left(J_{3}-r_{i} J_{1}\right)^{-1} B^{*}-A_{3}^{*}-\rho_{i} A_{5}^{*} .
$$

Так же как в разделе 4 , находится потенциал для представления $w_{i}^{*}$ :

$$
w_{i}^{*}=f^{*}-\left(\lambda / \partial_{i} \lambda\right) \partial_{i} f^{*}, \quad f^{*}=\pi^{-1} \int_{c}^{1} s \Gamma_{0}^{*}(s) \Delta(s) d s .
$$


Суммарный потенциал $f+f^{*}$ ( $f$ определяется формулой $\left.(34)\right)$ совпадает с решением $f_{\mathrm{II}}$ из работы $[10]$ (см. там формулу (15)). Для сравнения надо сделать замену $y=s^{2}$ и представить $\Gamma_{0}$ и $\Gamma_{0}^{*}$ через стандартный эллиптический интеграл $\mathrm{K}$.

Автор признателен А.В.Гуревичу и А.Л.Крылову, привлекшим его внимание к рассматриваемой проблеме, а также М.В.Павлову и Г.А.Элю за стимулируюший интерес к работе.

Автор благодарит Международный научный фонд за частичную поддержку настоящего исследования (грант RIE000).

\section{ПРИЛОЖЕНИЕ}

\section{Выражения через стандартные интегралы К, Е и П}

Полные эллиптические интегралы в нормальной форме [20]:

$$
\begin{gathered}
\mathrm{K}(m)=\int_{0}^{1}\left(1-t^{2}\right)^{-1 / 2}\left(1-m t^{2}\right)^{-1 / 2} d t, \quad \mathrm{E}(m)=\int_{0}^{1}\left(1-t^{2}\right)^{-1 / 2}\left(1-m t^{2}\right)^{1 / 2} d t \\
\Pi(n, m)=\int_{0}^{1}\left(1+n t^{2}\right)^{-1}\left(1-t^{2}\right)^{-1 / 2}\left(1-m t^{2}\right)^{-1 / 2} d t
\end{gathered}
$$

Для указанных в основном тексте величин получены следующие выражения:

$$
\begin{gathered}
J_{1}=d^{-1} \mathrm{~K}, \quad J_{3}=d^{-1}\left(c^{2} \mathrm{~K}-d^{2} \mathrm{E}\right) \\
J_{5}=d^{-1}\left[\left(2 a^{2} c^{2}-a^{4}+d^{4}(2+m) / 3\right) \mathrm{K}-2\left(a^{2} d^{2}+d^{4}(1+m) / 3\right) \mathrm{E}\right] .
\end{gathered}
$$

Здесь обозначено $d=\left(c^{2}-a^{2}\right)^{1 / 2}$ и аргументом в $\mathrm{K}(m), \mathrm{E}(m)$ является $m=$ $\left(b^{2}-a^{2}\right) d^{-2}$. Далее,

$$
\begin{gathered}
\Gamma_{0}(s)=2\left(M-a^{2}\right)^{-1 / 2}\left(c^{2}-\mu\right)^{-1 / 2} \mathrm{~K}\left(m^{*}\right), \\
\Gamma_{1}(s)=2\left(M-a^{2}\right)^{-1 / 2}\left(c^{2}-\mu\right)^{-1 / 2}\left[c^{2} \mathrm{~K}\left(m^{*}\right)-\left(c^{2}-a^{2}\right) \Pi\left(n, m^{*}\right)\right], \\
\beta(s)=2\left[\frac{\left(c^{2}-a^{2}\right)}{\left(M-a^{2}\right)\left(c^{2}-\mu\right)}\right]^{1 / 2}\left[\left(\frac{2 M+c^{2}-3 s^{2}}{3\left(c^{2}-M\right)}\left(c^{2}-b^{2}\right) \mathrm{K}(m)+\right.\right. \\
\left.\left.+\left(s^{2}-\frac{1}{2} \sigma\right) \mathrm{E}(m)\right) \mathrm{K}\left(m^{*}\right)+\left(c^{2}-b^{2}\right) \frac{\left(s^{2}-c^{2}\right)\left(M-a^{2}\right)}{\left(c^{2}-M\right)\left(c^{2}-a^{2}\right)} \mathrm{K}(m) \mathrm{E}\left(m^{*}\right)\right],
\end{gathered}
$$

где

$$
\begin{gathered}
M=\max \left(s^{2}, b^{2}\right), \quad \mu=\min \left(s^{2}, b^{2}\right), \quad \sigma=a^{2}+b^{2}+c^{2}, \\
m^{*}=\left(c^{2}-M\right)\left(\mu-a^{2}\right)\left(M-a^{2}\right)^{-1}\left(c^{2}-\mu\right)^{-1}, \quad n=\left(\mu-a^{2}\right)\left(c^{2}-\mu\right)^{-1} .
\end{gathered}
$$

С помошью вышеприведенных выражений для $J_{n}$ формулы (29) преобразуются к виду

$$
\begin{aligned}
& v_{1}=2\left(r_{1}+r_{2}+r_{3}\right)-4\left(r_{2}-r_{1}\right) \mathrm{K}(\mathrm{K}-\mathrm{E})^{-1}, \\
& v_{2}=2\left(r_{1}+r_{2}+r_{3}\right)-4\left(r_{2}-r_{1}\right)(1-m) \mathrm{K}(\mathrm{E}-(1-m) \mathrm{K})^{-1}, \\
& v_{3}=2\left(r_{1}+r_{2}+r_{3}\right)+4\left(r_{3}-r_{1}\right)(1-m) \mathrm{KE}^{-1}
\end{aligned}
$$




\section{Список литературы}

[1] Whitham G.B. // Proc.Roy.Soc.A. 1965. V. 283. P. 238.

[2] Гуревич А.В., Питаевский Л.П. // ЖЭТФ. 1973. Т. 65. С. 590.

[3] Гуревич А.В., Крылов А.Л., Мазур Н.Г. // ЖЭТФ. 1989. Т. 95. С. 1674.

[4] Гуревич А.В., Крылов А.Л., Мазур Н.Г., Эль Г.А. // ДАН СССР. 1992. Т. 323. С. 876.

[5] Царев С.П. // ДАН СССР. 1985. Т. 282. С. 534.

[6] Дубровин Б.А., Новиков С.П. // УМН. 1989. Т. 44. С. 29.

[7] Гуревич А.В., Крылов А.Л., Эль Г.А. // Письма в ЖЭТФ. 1991. Т. 54. С. 104.

[8] Кудашев B.P., Шарапов C.E. // ТМФ. 1991. Т. 87. С. 40.

[9] Гуревич А.В., Крылов А.Л., Эль Г.А. // ЖЭТФ. 1992. Т. 101. С. 1797.

[10] Крылов А.Л., Ходоровский В.В., Эль Г.А. // Письма в ЖЭТФ. 1992. Т. 56. С. 325.

[11] Tian F.R. // Commun.Pure Appl.Math. 1993. V. 46. P. 1093.

[12] Lax P.D., Levermore C.D. // Commun.Pure Appl.Math. 1983. V. 36. P. 253-290; 571-593; 809-830.

[13] Venakides S. // Commun.Pure Appl.Math. 1985. V. 38. P. 125.

[14] Venakides S. // Commun.Pure Appl.Math. 1990. V. 43. P. 335.

[15] Бикбаев Р.Ф. // Функц.анализ и его прилож. 1989. Т. 23. С. 1.

[16] Bikbaev R.F. // Phys.Lett.A. 1989. V. 141. P. 289.

[17] Геогджаев В.В. // ДАН СССР. 1985. Т. 284. С. 1093.

[18] Солитоны / Ред. Р.Буллаф, Ф.Кодри. М.: Мир, 1983. С. 175-192.

[19] Лаврентьев М.A., Шабат Б.В. Методы теории функций комплексного переменного. М.: Физматгиз, 1958. С. 284-288.

[20] Янке E., Эмде Ф., Леш Ф. Специальные функции. М.: Наука, 1968.

Институт физики Земли

Поступила в редакцию им. О.Ю.Шмидта 17.III.1995г

Российской академии наук

\section{N.G. Mazur \\ QUASICLASSICAL ASYMPTOTICS OF THE INVERSE SCATTERING SOLUTIONS OF THE KdV EQUATION AND THE SOLUTION OF WHITHAM'S MODULATION EQUATIONS}

The initial value problem for the $\mathrm{KdV}$ equation is studied in the limit of weak dispertion. It may be considered as a model for nondissipative shock wave in plasmas. The perturbation theory in power series of the weak dispersion parameter leads to the Riemann simple wave equation describing nonlinear effects of wave front sharpening and "overturning". The subsequent phase of the nondissipative shock wave evolution is described by Whitham's modulation equations.

Another approach used in this paper is based on the asymptotic study of the exact solution by the inverse scattering problem technique. The WKB formulas for the direct scattering problem solution and the exact multisolution of the inverse problem are considered. As a result a system of closed relations between $x, t$ and the modulation parameters is obtained, which presents an exact solution of the Whitham's equations. 